\title{
APORTE DE LAS ESTRATEGIAS DE MINDFULNESS A LOS COMPONENTES DE AUTORREGULACIÓN DEL APRENDIZAJE DE LOS ESTUDIANTES DE PREESCOLAR
}

Contribution of mindfulness strategies to the learning's self-regulation components of preschool students

Contribuição de estratégias de mindfulness para os componentes de auto-regulação da aprendizagem de pré-escolares

\section{Francisco Conejo Carrasco}

Corporación Universitaria Minuto de Dios, Colombia

francisco.conejo@uniminuto.edu

\author{
Jenny Consuelo Mahecha Escobar \\ Corporación Universitaria Minuto de Dios, Colombia \\ jmahecha@uniminuto.edu \\ Oxana Cerra Guerra \\ ocerraguerr@uniminuto.edu.co
}

Resumen

El presente artículo presenta los resultados de una investigación que buscaba analizar la influencia que ejercen las estrategias Mindfulness en el desarrollo de los componentes de la autorregulación del aprendizaje de los estudiantes de grado jardín del Colegio Nueva York, en Bogotá, Colombia. A través de un método cualitativo con diseño descriptivo se evidencia un entendimiento de los fenómenos desde el punto de vista docente, al trabajar con una muestra de 9 estudiantes y 3 docentes. Los datos recogidos por medio de la rejilla de observación, entrevista a docentes y diario de campo fueron analizados permitiendo establecer que los estudiantes con mayores dificultades de atención y acciones disruptivas fueron los que mostraron una mejoría con la implantación en sus factores de autorregulación del aprendizaje. La implementación de espacios metacognitivos en estudiantes de temprana 
edad fomenta la autorreflexión y promueve los procesos de autoconciencia a través de estrategias de Mindfulness.

Palabras clave: conciencia plena; autorregulación del aprendizaje; preescolar; cognición; emoción; conducta

\begin{abstract}
This article presents the results of an investigation that seeks to analyze the influence that Mindfulness strategies exert on the development of the components of the self-regulation of learning of the undergraduate students of the New York College, in Bogotá, Colombia. Through a qualitative method with descriptive design, an understanding of the phenomena from the teaching point of view is evidenced, working with a sample of 9 students and 3 teachers. The data collected through the observation grid, teacher interview and field diary were analyzed to establish which students with greater attention difficulties and disruptive actions were those who improved with the implementation of their self-regulation factors of learning. The implementation of metacognitive spaces in young students encourages selfreflection and promotes self-awareness processes through Mindfulness strategies.
\end{abstract}

Key words: mindfulness; self-regulation of learning; preschool; cognition; emotion; behavior

\title{
Resumo
}

Este artigo apresenta os resultados de uma investigação que busca analisar a influência que as estratégias de Mindfulness exercem no desenvolvimento dos componentes da autoregulação da aprendizagem de estudantes de graduação do New York College, em Bogotá, Colômbia. Por meio de um método qualitativo, com desenho descritivo, evidencia-se a compreensão dos fenômenos do ponto de vista do ensino, trabalhando com uma amostra de 9 alunos e 3 professores. Os dados coletados por meio da grade de observação, entrevista com professor e diário de campo foram analisados para estabelecer quais alunos com maiores dificuldades de atenção e ações disruptivas foram os que melhoraram com a implementação de seus fatores de aprendizagem de auto-regulação. A implementação de espaços 
metacognitivos em jovens estudantes incentiva a auto-reflexão e promove processos de autoconsciência por meio de estratégias de Mindfulness.

Palavras chave: atenção plena; auto-regulação da aprendizagem; pré-escola; cognição; emoção; comportamento

\section{Introducción}

En este trabajo se ha investigado el impacto que pueden llegar a tener algunas estrategias de Mindfulness en los componentes de la autorregulación en los niveles iniciales de la educación. Con esto en mente, surge la necesidad de abordar las problemáticas educativas de forma integral, entendiendo al ser humano como el propio generador de su desarrollo por medio de las herramientas que adquiere de su contexto. Además, al tener en cuenta que los primeros años de vida son esenciales para el desarrollo del ser humano ya que las experiencias tempranas perfilan la arquitectura del cerebro y diseñan el futuro comportamiento (Campos, 2010), se justifica la implementación de las estrategias Mindfulness y las investigaciones al respecto, desde el nivel preescolar.

Para el presente estudio, se tomó en consideración la problemática particular del Colegio Nueva York, el cual es un colegio privado mixto ubicado en el norte de la ciudad de Bogotá que cuenta con los niveles de Preescolar, Básica y Media. La filosofía pedagógica de la institución es altamente constructivista con fuertes influencias de la pedagogía sistémica y la afectiva, donde el estudiante de preescolar es entendido como un individuo único, con una increíble capacidad creativa y curiosidad, que lo llevan a descubrir las maravillas del mundo que lo rodea. De esta forma, el docente es un facilitador de experiencias que priorizan las relaciones y el aprendizaje en diferentes entornos.

Sin embargo, los grupos en el colegio son numerosos, de aproximadamente 20 a 25 estudiantes en el preescolar. Esto es un factor significativo que dificulta a los docentes el proceso de captar y mantener la atención de sus alumnos en cada actividad y entorpece el trabajo diferenciado con cada estudiante. Al realizar un sondeo exploratorio previo, muchos profesores expresan utilizar estrategias conductistas, las cuales ofrecen resultados insuficientes que no funcionan para todos. Es así que, se observa una necesidad de estrategias 
que permitan fortalecer los procesos autorregulatorios de los estudiantes y que impliquen una transformación real de los métodos utilizados a largo plazo en el ámbito educativo. Teniendo en cuenta que, las estrategias de Mindfulness o conciencia plena han demostrado contribuir a la capacidad para mejorar problemas de salud en adultos y apoyarlos en el manejo del estrés (De Vibe, Bjørndal, Fattah, Dyrdal, Halland, \& Tanner- Smith, 2018), se proponen como eje de intervención de este proyecto. Consecuentemente, el propósito de este estudio fenomenológico es dar respuesta a ¿Cómo influyen las estrategias de Mindfulness o conciencia plena en el desarrollo de los componentes de la autorregulación del aprendizaje en los estudiantes de Jardín del Colegio Nueva York?

Entonces, este artículo contiene el fundamento teórico que sustenta toda la investigación realizada. Aquí se interioriza en los conceptos de autorregulación, examinando su desarrollo y sus componentes, así como también en la definición de Mindfulness y sus prácticas. A continuación, se hace referencia a la metodología utilizada, donde se relatan las estrategias a seguir para alcanzar los objetivos propuestos. Finalmente, se enuncian y analizan los resultados obtenidos de acuerdo a cada una de las categorías y subcategorías, así como también se presentan las conclusiones finales.

\section{La autorregulación del aprendizaje}

En el contexto del aprendizaje, cada sujeto es capaz de adaptar sus funciones de acuerdo a las necesidades, de forma que ejecuten las tareas correctamente o que corrijan las que no están siendo llevadas a cabo de forma apropiada, lo que se conoce como autorregulación. En este sentido, los estudiantes deben ser capaces de identificar y modificar las falencias que puedan tener en algún área (Vives, Durán, Varela, \& Fortoul, 2014). Es así que, la autorregulación se refiere al control que puede llegar a tener un estudiante sobre su cognición, comportamiento y emociones, el cual se lleva a cabo a través de diferentes estrategias que lo ayudan a materializar sus objetivos (Alonso y Panadero, 2014), dichas estrategias son susceptible de ser aprendidas a través del entrenamiento adecuado.

\section{a. La metacognición y las funciones ejecutivas}


La metacognición se encarga de controlar la capacidad de adquirir conocimiento y durante mucho tiempo fue considerada como el único proceso que participaba en el aprendizaje (Alonso y Panadero, 2014). Es así que, para autorregularse el individuo debe utilizar una serie de tácticas cognitivas y de esta forma poder realizar las actividades requeridas. En este sentido, se puede afirmar que, las estrategias metacognitivas son aquellas técnicas que facilitan el desarrollo de los procesos de planeación, ejecución, supervisión y evaluación del desempeño propio al realizar una tarea (Gaeta, Pilar y Orejudo, 2012). Dichas estrategias, también conocidas como Funciones Ejecutivas (FE) envuelven los procesos mentales que intervienen para solucionar las situaciones de la vida diaria (Juric, Introzzi, Andrés y Stelzer, 2016).

Entre las Funciones Ejecutivas de orden superior se encuentran "la memoria de trabajo, la planeación, organización, la fluidez, flexibilidad, automonitoreo, control inhibitorio y la atención" (Batista, 2012, p.6), todas relacionadas directamente con el proceso autorregulatorio. Sin embargo, para efectos de esta investigación se hizo hincapié en la última por su conexión con el problema a resolver. La atención se ha definido como el sistema jerárquico de funciones que dirige la percepción de los estímulos que realmente necesita e inhibe los no deseados, de esta forma se activa el procesamiento de la información recibida (Farah, 2000; Posner, 1980; Roselló Mir, 1998; citados en Monteolivo et al., 2014). Por lo tanto, implica interesarse por un estímulo dirigiendo la gestión cerebral hacia él, buscando conocerlo mejor. La misma, se relaciona además con el autocontrol, la conciencia social y la capacidad de adaptación (Ruff y Rothbart, 1996; citados en Pérez, 2009).

Por estos motivos, los estudios realizados sobre el tema permiten definir tres procesos principales para la atención: inicio, mantenimiento y cese. Según esta teoría en un principio se recogen datos iniciales acerca de los aspectos más llamativos de un estímulo como color, forma, tamaño e intensidad, luego se mantienen dirigidos los sentidos hacia el estímulo con el objetivo de recopilar más información, lo que puede durar varios minutos dependiendo de la edad del sujeto; finalmente la atención se dirige hacia otro estímulo, lo que provoca que se pierda la concentración en el anterior (Flores, 2016). De acuerdo a los estudios realizados se calcula que los niños y niñas de 5 años pueden mantener su atención visual en una actividad 
por 14 minutos aproximadamente. En el nivel preescolar, la atención está ceñida a diversos aspectos como la hora, la complejidad y el tipo de tarea o actividad, los intereses de cada niño, entre otros. Sobre los 5 años de edad los niños se vuelven capaces de conducir su atención hacia los elementos de su interés y de ignorar aquellos que no lo son, llegando incluso a modificar su foco atencional dentro de un mismo estímulo (Pérez, 2009).

\section{b. Las emociones}

Se puede definir la emoción como una respuesta que se manifiesta de forma psicológica y fisiológica ante un fenómeno determinado, que tiene su origen en la teoría de la evolución y han permitido la adaptación del ser humano a la sociedad (Castillo, 2013). Tal como afirman las investigaciones en neurociencias, las emociones pueden potenciar o bloquear el aprendizaje, de ahí la importancia de controlarlas (Alonso y Panadero, 2014).

Goleman (1995) acuñó el término Inteligencia Emocional (IE) para referirse a la capacidad que tiene el ser humano de controlar sus propios sentimientos por medio del desarrollo de habilidades que son susceptibles de aprenderse. Entre los 4 y los 5 años, los niños comienzan a experimentar el desarrollo del control externo -proveniente de padres y maestros- al interno, lo que provoca respuestas poco favorables a nivel conductual en el ambiente educativo. La regulación emocional es la habilidad más difícil de alcanzar ya que implica contener las respuestas naturales a los estímulos en situaciones que se salen de nuestro control. En consecuencia, la enseñanza de técnicas para el manejo de la ira, retraso de gratificaciones y el aumento de la tolerancia ante la frustración son habilidades necesarias para una correcta autorregulación (Moctezuma, 2016).

\section{c. El comportamiento}

El control del comportamiento es un proceso primordial dentro de la autorregulación ya que las acciones son las que llevan al estudiante a alcanzar los objetivos propuestos (Alonso y Panadero, 2014). Está demostrado que las cualidades propias de la conducta prosocial como son la empatía, disciplina y autocontrol se corresponden con el buen desempeño académico (Gallegos, 2015). Es así que, desarrollar una conducta prosocial contribuiría al proceso autorregulatorio y este a su vez, permitiría mayor control de los comportamientos. 
Los estudios que investigan el comportamiento estudiantil se han encaminado hacia una metodología experimental, de acuerdo a los diferentes enfoques conductuales. El enfoque de modificación de conducta, a pesar de mostrar muy buenos resultados, no muestra continuidad una vez terminada la intervención; mientras que el enfoque de modificación de la conducta cognitiva no muestra resultados en el medio educativo, en definitiva, aquí no se está entendiendo al niño como un todo, sino que se analizan y clasifican sus conductas de forma superficial (Bazán, 2017). Lo que sí se ha logrado establecer es que, la insuficiencia en cualquier proceso autorregulatorio puede provocar discrepancias entre las intenciones o actitudes y el comportamiento observable (Kuhl, 1985).

Además, han podido relacionar el temperamento con el comportamiento en la infancia sugiriendo que el control intencional es la principal dimensión asociada a las conductas disruptivas y la agresividad. De esta forma, autorregular la reactividad comportamental implica que la potencia y persistencia de una conducta indeseada sea cada vez menor (Bárrig Jo y Alarcón Pardo, 2017). Por otra parte, se ha demostrado que la manifestación de conductas disruptivas en la infancia puede revelar comportamientos antisociales en edades más avanzadas (Alonso, Vergara, Gutiérrez y Vozmediano, 2017). Con esto se demuestra la importancia de tomar en cuenta los procesos mentales en la implementación de estrategias orientadas a la regulación comportamental.

\section{Práctica del Mindfulness}

El significado de la palabra Mindfulness se remonta a la lengua pali, donde abarca la conjunción de los conceptos de conciencia, atención y recordar, con lo cual se hace referencia al estado mental en donde se recuerda siempre prestar atención y se es consciente del momento presente (Amigo y Gonzáles, 2017). Es así que algunos autores conceptualizan al Mindfulness como una actitud que consiste en tomar en cuenta los pensamientos y emociones que se perciben en cada momento, esto implica que el individuo que lo practica debe autorregular sus procesos cognitivos, emocionales y conductuales. Por otra parte, se puede hablar de Mindfulness como una práctica permanente que, una vez dominada e interiorizada conduce a un rasgo de la personalidad o estado mental permanente conocido como conciencia plena (Hervás, Cebolla y Soler, 2016). A pesar de no haber un consenso en cuanto a la 
conceptualización si existen dos componentes claves que derivan del término: el comportamiento y cómo este se guía hacia un estado deseado (Delgado, Guerra, Perakakis y Viedma, 2010).

Estudios recientes demuestran que los beneficios de la práctica del Mindfulness comprenden un incremento en el control de los procesos atencionales, el dominio de habilidades para regular las emociones y el aumento de la autoestima (Hervás, Cebolla y Soler, 2016). De igual forma, se ha concluido que, por medio de la práctica de la atención plena se pueden modificar las respuestas involuntarias a ciertos estímulos al autorregular la forma en la que se experimentan las emociones que derivan impulsivamente. Por lo tanto, esta práctica busca que el individuo preste atención y deje fluir las sensaciones que percibe, acoja los pensamientos y las emociones sin juzgarlos. Esto permite disminuir el estrés provocado por el miedo y otras emociones negativas provenientes de evaluar constantemente lo que se siente o se piensa (Arias, Granados y Justo, 2010). En este orden de ideas, se puede afirmar que la práctica de la conciencia plena constituye un método de autorregulación de las emociones y el comportamiento.

Finalmente, a nivel mundial se evidencian experiencias transversales en el tiempo como los programas TREVA (Técnicas de Relajación Vivencial Aplicadas al Aula) y Aulas felices que están siendo implementados con gran acogida en colegios de España. De dichas experiencias se extrajeron algunas técnicas para practicar el Mindfulness pertinentes para esta investigación, como son la relajación por medio de la respiración consciente, el escaneo corporal, la meditación, entre otras.

\section{Observando las prácticas en el preescolar}

Para esta investigación se propuso un diseño metodológico de tipo descriptivo donde, al analizar los datos, se puede establecer la diferenciación de un fenómeno de acuerdo a las peculiaridades de cada sujeto estudiado (Cardona, 2015), de forma que permitiera poner de manifiesto las características propias de la población estudiada y la repercusión que puedan o no tener las estrategias de Mindfulness en el desarrollo de habilidades autorregulatorias. 
Es así que, se seleccionó la muestra de forma intencional de acuerdo a las particularidades de los sujetos, escogiendo los participantes más idóneos que cumplan con unos criterios básicos, esto permitió conformar los dos grupos -estudiantes y docentes- que participaron de la investigación. En el caso de los estudiantes, los criterios de selección incluyeron su disposición para realizar la actividad, pero también se buscaba conformar un grupo heterogéneo de 9 estudiantes, de los cuales 4 fueron niñas y 5 niños, por lo que se agregaron algunos estudiantes que presentaban comportamientos disruptivos y otros que no. La muestra de docentes estuvo conformada 3 profesoras que dictan clase a la muestra de estudiantes.

La elaboración de instrumentos específicos para esta investigación permitió recopilar los datos concretos que respondieran a cada uno de los objetivos propuestos. Es así que, la entrevista semiestructurada, aplicada de forma individual a la muestra de docentes escogida, buscó contextualizar el problema y conocer las diferentes perspectivas de los profesionales acerca de las conductas observables de los estudiantes antes y después de haber asistido a una sesión Mindfulness. Se trató de una conversación fluida, con objetivos previamente establecidos, que propendía por recopilar información que sustente y responda la pregunta de investigación. Entonces, la entrevista, como primer instrumento, conformado por preguntas abiertas previamente estipuladas en un protocolo y las que surgieron durante la conversación, contribuyó a validar la efectividad de las estrategias propuestas.

Por otra parte, el diseño de la rejilla de observación concede al investigador los medios para que pueda enfocarse en la información más relevante. En este sentido, el análisis de la información recopilada para construir los antecedentes y el marco teórico de esta investigación permitió el diseño de una rejilla de observación que incluyera como indicadores dichos rasgos considerados repetitivos, la cual permitió obtener una descripción detallada de los comportamientos más característicos percibidos en los estudiantes durante el desarrollo habitual de sus clases, así como también, comprobar la repetición o disminución de dichas conductas después de aplicar la intervención.

El tercer instrumento consistió en un diario de campo que fue completado por la investigadora durante la observación de la segunda hora de clases, tanto en la primera semana como en la segunda. Dicho documento pretendía recoger información en cuanto al día, lugar 
y recursos necesarios para realizar cada actividad, así como también, las limitaciones y eventos especiales relevantes y que aportaron datos para resolver los objetivos propuestos. De esta forma se logró conjugar la teoría y la práctica para su análisis.

El trabajo de campo se dividió en tres fases, de acuerdo a los dos grupos de muestra seleccionados. La primera fase se basó en aplicar la entrevista a los docentes para conocer cómo son esos comportamientos que normalmente se aprecian en la muestra y observar la segunda hora de clases durante una semana, completando la rejilla de observación con respecto a los comportamientos observados en los estudiantes. La segunda fase consistió en aplicar 5 talleres de Mindfulness a la muestra de estudiantes durante la siguiente semana en la primera hora y observar nuevamente la siguiente hora de clase acopiando los datos por medio de la rejilla de observación previamente diseñada y validada. En este caso se tomaron videos de la ejecución para favorecer la recogida de datos más generales en el diario de campo. Finalmente, durante la tercera fase se realizaron las mismas entrevistas a los docentes, buscando establecer si observaban cambios en el comportamiento y en qué aspectos más que otros.

\section{Dar sentido a lo observado desde la propia perspectiva}

El primer objetivo específico buscaba identificar los rasgos cognitivos, conductuales y emocionales que normalmente evidencian en clase los estudiantes de preescolar. A partir de la observación inicial, se pudo establecer que, en la muestra estudiada, el rasgo cognitivo que más se aprecia es un ritmo atencional corto, aun para la edad preescolar. Esto incluye los tres procesos de la atención -inicio, mantenimiento y cambio o cese-, con una mayor incidencia de dificultades en el mantenimiento, tal como se evidencia en el diario de campo, donde se consigna que "La habilidad de mantener la atención en una misma actividad por al menos diez minutos es la que más se dificulta. Se observan 7 estudiantes que muestran inconvenientes relacionados con la pérdida de la concentración en la actividad que estaban realizando."

Por su parte, el rasgo emocional más desarrollado es el reconocimiento de las emociones, tanto las propias como las de otros. Con respecto al proceso de la empatía, es notorio su poco desarrollo en los estudiantes que participaron de la investigación, esto debido a su corta edad. 
Si bien los estudiantes fueron capaces de inferir qué estímulos han causado las emociones negativas (frustración y tristeza) en sus compañeros, no empatizaron con ellos a menos que les sucediera lo mismo, o sea, que perdieran un juego, por ejemplo. Esto se pudo triangular con los datos arrojados por la rejilla de observación, en la cual se observó un porcentaje de dificultad inicial del $59 \%$ en cuanto a la habilidad de la muestra para mostrarse empáticos.

Además, se logró establecer que el rasgo conductual que más se presenta es la dificultad para resolver conflictos. Esto depende en gran medida de las actividades propuestas, por eje $5 \mathrm{mplo}$, en el Diario de campo del 15 de octubre se consigna: “durante la visualización del video hablan e interrumpen por lo que se debe repetir la proyección. Se presentan conflictos aislados al querer hablar todos al tiempo durante la reflexión y se evidenciaron demostraciones de agresividad y frustración por tener que esperar turnos". Vale aclarar que esta habilidad se encuentra en una etapa de desarrollo debido al proceso de separación de la comprensión egocéntrica del mundo que tiene el niño en edad preescolar, de acuerdo a los postulados de Piaget.

A continuación, el segundo objetivo específico pretendía definir el tipo de estrategias Mindfulness que promueven los componentes de autorregulación de los estudiantes. De acuerdo a esto, no se logró se establecer la efectividad de las estrategias RAIN y respiración -por sí sola- ya que los datos recogidos en los instrumentos no fueron concluyentes, lo cual tiene relación con el poco tiempo de ejecución, por lo que infiere que se requiere implementar más sesiones para poder apreciar una diferencia en los comportamientos.

Figura 1 Dificultades generales en los tres componentes, previo y posterior a la implementación de la estrategia Yoga. Fuente: Elaboración propia (2019).

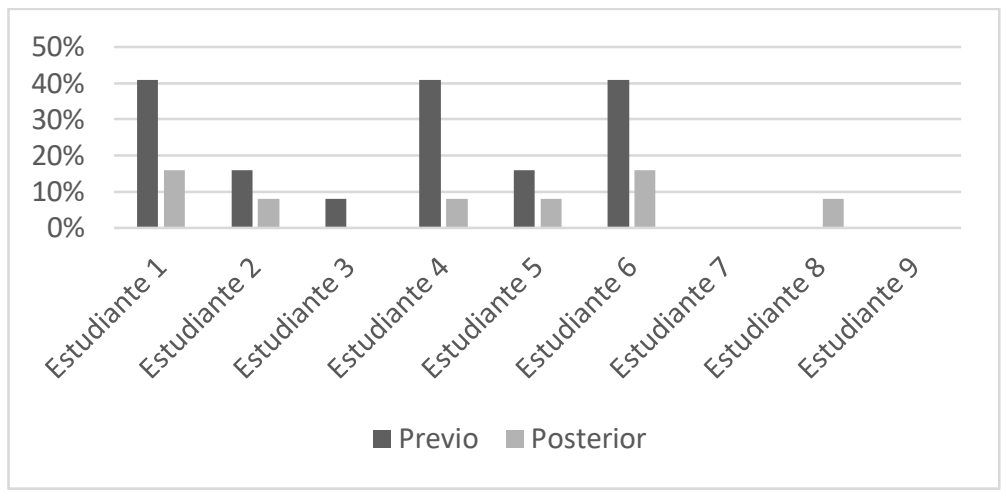


Por su parte, las estrategias Yoga y meditación demostraron un alto impacto, sin embargo, se relacionan con mejorías en diferentes componentes de la autorregulación. Es así que el Yoga impactó principalmente el componente cognitivo mientras que la meditación demostró alta efectividad en los componentes emocional y conductual, tal como se evidencia en las figuras 1 y 2.

Figura 2 Dificultades generales en los tres componentes, previo y posterior a la implementación de la estrategia Meditación. Fuente: Elaboración propia (2019).

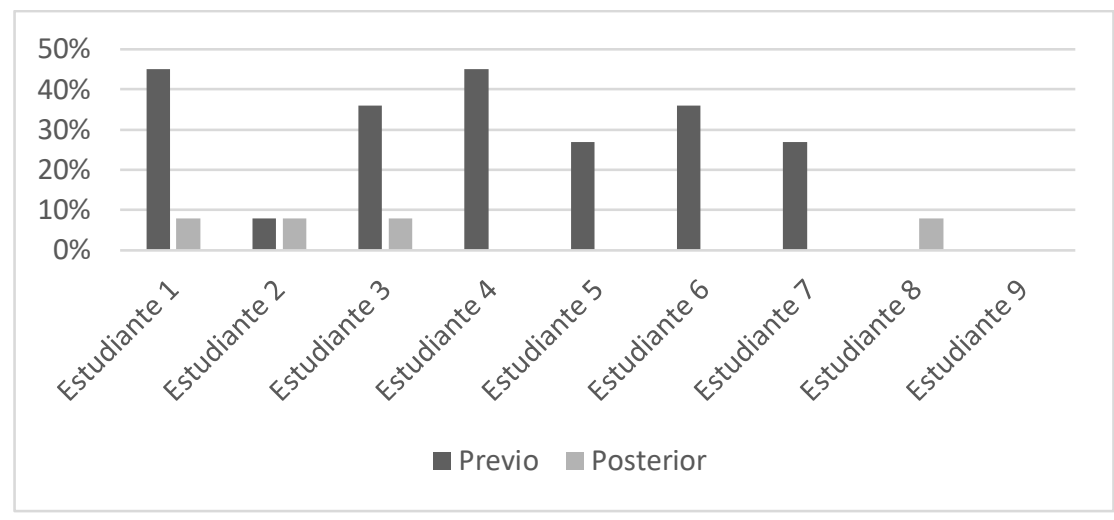

Finalmente, el tercer objetivo específico buscaba describir los componentes de la autorregulación que se impactan con la aplicación de estrategias basadas en Mindfulness. Según los datos obtenidos, en los tres componentes estudiados -cognitivo, emocional y conductual- se aprecian beneficios a partir de las estrategias implementadas, aunque en diferente medida. En cuanto al componente cognitivo, es evidente la disminución de dificultades para trasladar la atención a un nuevo estímulo, ya que no se evidenciaron anotaciones en este proceso durante la observación posterior. De igual forma, el análisis estadístico, mostrado en las figuras 3 y 4, expone menores porcentajes de problemáticas en el inicio y finalización de la atención en los estímulos presentados, lo cual se corresponde con lo percibido por las docentes en las entrevistas realizadas.

En este sentido, se entiende su influencia para facilitar los procesos atencionales al preparar el cerebro para la concentración y el aprendizaje, lo cual se evidencia en lo expresado por la docente 3: "Ellos necesitan que su cerebrito esté preparado para recibir todos los estímulos que día a día a nosotros les traemos a clase". Esto se relaciona con lo expresado por Pérez (2009) cuando aduce que la atención es reconocida como uno de los mecanismos que 
permiten el funcionamiento del sistema nervioso, junto con el motor y el sensorial, de forma que se hace referencia a la atención como el sistema que posibilita procesar la información y a su vez como a los procesos cerebrales que la engloban, por lo que se infiere aquí que lo señalado por las docentes es pertinente.

Figura 3 Dificultades en el componente cognitivo por estudiante durante la observación previa. Fuente:

Elaboración propia (2019).

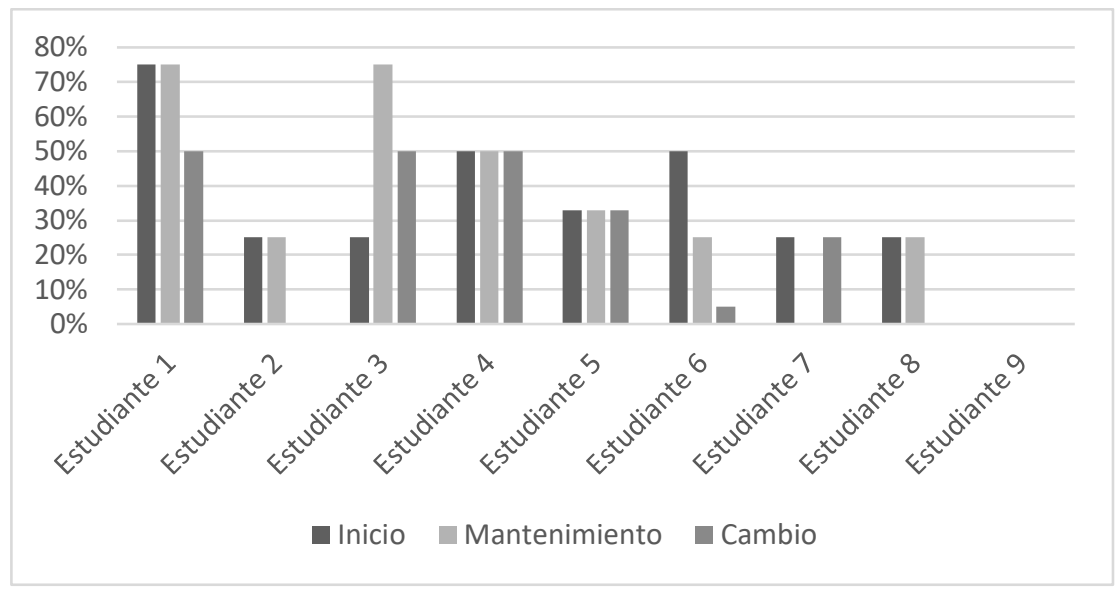

Cabe resaltar aquí lo expresado por Jiménez, Hernández, García, Díaz, Rodríguez y Martín (2012) que conceptualizan la atención selectiva como la habilidad de concentrarse en uno o dos elementos, inhibiendo los demás estímulos que se reciban -relacionada con el cambio en el foco de atención-, y la atención sostenida como la capacidad de mantener la concentración en un mismo estímulo por un largo periodo de tiempo -asociada con el mantenimiento de la atención-.

Figura 4 Dificultades en el componente cognitivo por estudiante durante la observación posterior. Fuente:

Elaboración propia (2019).

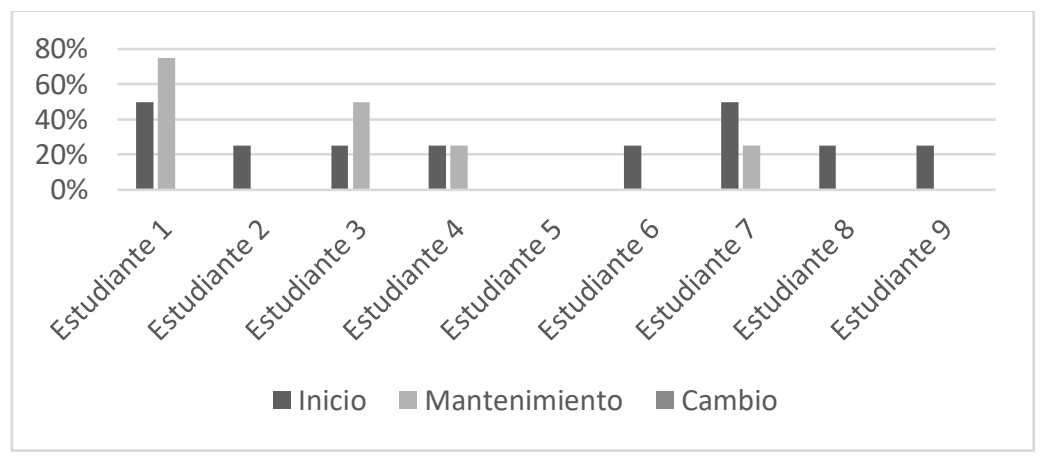

Aporte de las estrategias de mindfulness a los componentes... 
Entonces, se entiende que si bien, las estrategias Mindfulness pudieron impactar positivamente en la atención selectiva, no se encuentran evidencias de su influencia en la mejora de los tiempos de atención sostenida de la muestra estudiada.

Por otra parte, para el análisis del componente emocional se tomaron en cuenta los procesos reguladores de las emociones en los estudiantes del curso Jardín, teniendo en cuenta las capacidades según la edad y etapa de la vida en la que se encuentran. En este orden de ideas se indagó acerca de la capacidad para reconocer las emociones propias, las de otros individuos y la habilidad de ser empáticos, o sea, simular las emociones que pueden estar sintiendo sus compañeros en determinado momento.

Figura 5 Dificultades en el componente emocional por estudiante durante la observación previa. Fuente:

Elaboración propia (2019).

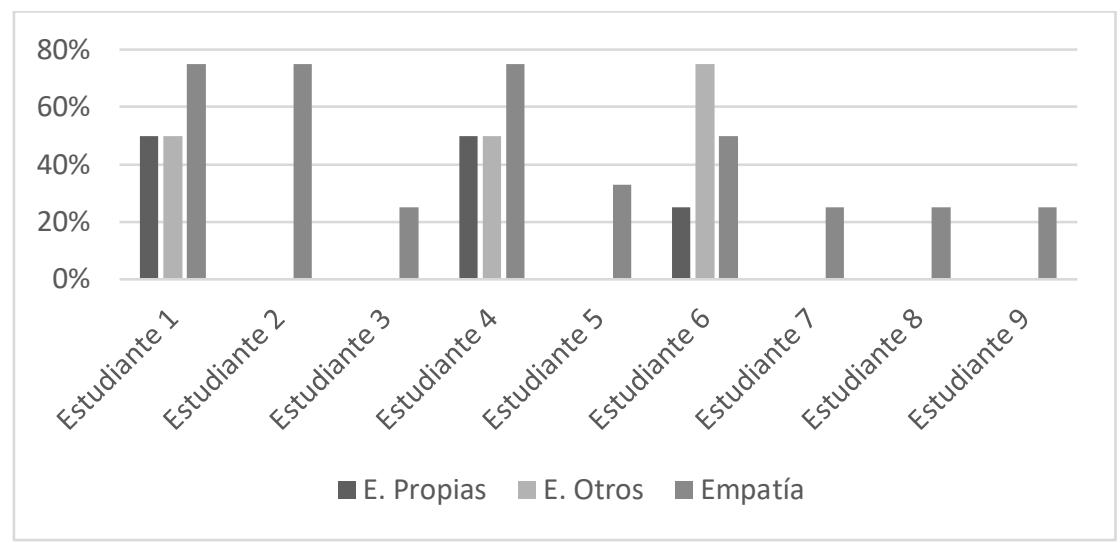

Figura 6 Dificultades en el componente emocional por estudiante durante la observación posterior. Fuente: Elaboración propia (2019).

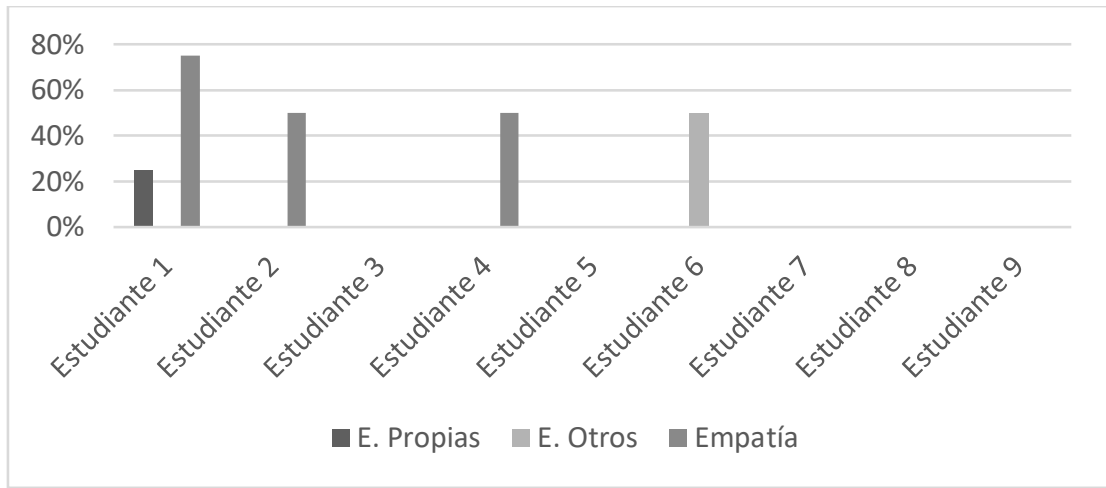


Teniendo en cuenta los datos obtenidos en la rejilla de observación que se observan en las figuras 5 y 6 , se infiere que las estrategias implementadas tuvieron un fuerte impacto en el componente emocional, demostrando que la enseñanza de técnicas para el manejo de la ira, retraso de gratificaciones y el aumento de la tolerancia ante la frustración, incluidas en la estrategia RAIN, son necesarias para una correcta autorregulación emocional.

Finalmente, para el análisis del componente conductual se describen los comportamientos observados en cuanto a las manifestaciones de agresividad, la identificación de conductas disruptivas y la resolución de conflictos. Se observa que los estudiantes que demostraron conductas agresivas durante las observaciones previa y posterior fueron los mismos, aunque se aprecia una leve disminución de estos comportamientos.

Figura 7 Dificultades en el componente conductual por estudiante durante la observación previa. Fuente:

Elaboración propia (2019).

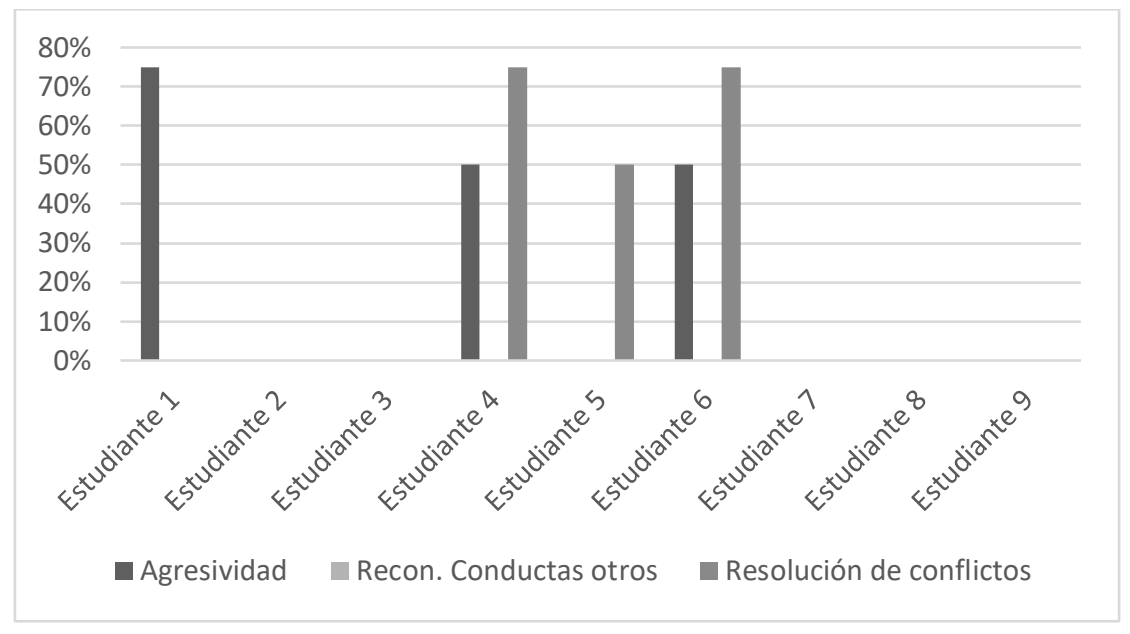

Es así que, se evidencia en los hallazgos del componente conductual que los comportamientos observados son susceptibles de ser modificados de forma positiva, indicando que este componente fue levemente impactado por las estrategias implementadas, como se aprecia en las figuras 7 y 8 . Esto es coherente con las respuestas de las docentes en torno a este componente, las cuales demuestran un consenso en cuanto a que los estudiantes se mantienen más calmados después de ejecutar ejercicios relacionados al Mindfulness y buscan formas efectivas para resolver los problemas, lo que se evidencia en el comentario de 
la Docente 2: "Por la misma dinámica del trabajo en grupo vimos algunos conflictos pero también ellos intentaron resolverlos de forma pacífica sin pelear ni agredir”.

Figura 8 Dificultades en el componente conductual por estudiante durante la observación posterior. Fuente:

Elaboración propia (2019)

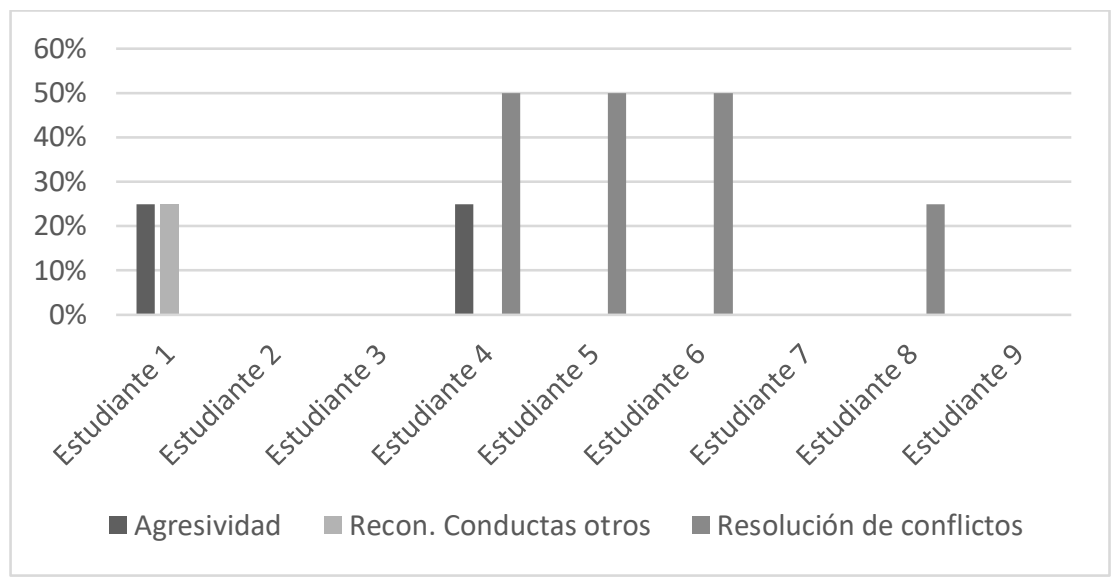

\section{Conclusiones}

El componente cognitivo es el que presentó unas dificultades más evidentes durante la observación previa. Sin embargo, a partir de la primera aplicación de las estrategias Mindfulness se pueden apreciar resultados favorecedores en su desarrollo, lo cual constituye un hallazgo relevante en el ámbito educativo, por ser una problemática recurrente en este contexto.

Para poder percibir cambios en el componente emocional en los niños de corta edad es preciso contar con actividades que requieran la interacción entre pares y poder hacer un seguimiento a través del tiempo. Esto es un aspecto relevante a tener en cuenta en todas las investigaciones que busquen establecer hallazgos con respecto a lo emocional ya que no siempre se exteriorizan verbalmente, sino que requiere de una observación más profunda y prolongada para distinguirlos.

Las conductas disruptivas fueron observadas de forma recurrente en ciertos estudiantes, lo que, según los referentes consultados, está asociado al temperamento propio de cada individuo. Además, se aprecian dificultades en la muestra estudiada para reconocer este tipo de conductas en el proceder propio mientras son fácilmente identificadas cuando sus 
compañeros las cometen. La trascendencia de este descubrimiento radica en la necesidad de enfocar los esfuerzos hacia la observación de los comportamientos propios, entendida como una mirada hacia el interior, destreza que se fortalece con la práctica del Mindfulness.

Los estudiantes con mayores dificultades en todos los componentes durante la observación inicial fueron los que mostraron una mejoría más evidente en todos los componentes. Este hallazgo es uno de los más relevantes por cuanto confirma que la práctica de estrategias Mindfulness aporta beneficios a todos los estudiantes, pero en mayor medida a aquellos que más lo necesitan, reafirmándose como una herramienta ideal para el docente contemporáneo. Como principal resultado se planteó proponer una estrategia basada en Mindfulness que promueva el proceso de autorregulación en los niños de preescolar del Colegio Nueva York. Tomando en consideración que dichas estrategias impactan positivamente en los tres componentes de la autorregulación, siempre y cuando se ejecuten de forma periódica y cuenten con unos propósitos claros, se hace pertinente el diseño de un programa de estrategias de Mindfulness para ser implementado en todo el preescolar.

Por último, se espera que este estudio permita abrir nuevas posibilidades de investigación sobre el desarrollo de la autorregulación en los primeros años de vida, destacando que, por medio de la observación y con el apoyo de instrumentos específicamente diseñados, se pueden obtener resultados reveladores, aun cuando a la muestra no está lista para expresar verbalmente toda su percepción de las situaciones en las que se desenvuelve. Es aquí donde se hace pertinente un diseño metodológico enfocado en la didáctica necesaria para llegar a apreciar las situaciones y realidades de los estudiantes del nivel preescolar, enriqueciendo así el conocimiento acerca del desarrollo de la autorregulación desde la infancia.

\section{Referencias bibliográficas}

Alonso, J. \& Panadero, E. (2014). ¿Cómo autorregulan nuestros alumnos? Modelo de Zimmerman sobre estrategias de aprendizaje. Anales de Psicología / Annals of Psychology, 30(2), 450-462. 
Alonso, N., Vergara, A., Gutiérrez, J., \& Vergara, L. (2017). Conocimiento emocional y conducta disruptiva: evidencia preliminar para la prevención temprana de la conducta antisocial. International E-Journal of Criminal Sciences, (11), 3.

Amigo, I., \& González, G. (2017). Efectos de la atención plena en el ámbito educativo: una revisión sistemática. Revista de Psicología y Educación - Journal of Psychology and Education, 13(1), 63.

Arias, M. de la F., Granados, M. S., \& Justo, C. F. (2010). Efectos de un programa de entrenamiento en conciencia plena (Mindfulness) en la autoestima y la inteligencia emocional percibida. S - ProQuest. Psicología Conductual, 2, 297-315.

Bárrig Jó, P., \& Alarcón Parco, D. (2017). Temperamento y competencia social en niños y niñas preescolares de San Juan de Lurigancho: un estudio preliminar. Liberabit, 23(1), 75-88.

Batista, J. (2012). Las Funciones Ejecutivas. LUMEN, 14, 10.

Bazán, J. A. R. (2017). Autorregulación en preescolares con problemas de conducta. Congreso Nacional de Investigación Educativa, 14, 10.

Calventus, J. (2008). Una aproximación al análisis de datos cualitativos textuales. Valparaíso (Chile): Universidad de Valparaíso.

Campos, A. (2010). Primera infancia: Una mirada desde la neuroeducación. OEA/OEC.

Cardona, J. (2015). Ortodoxia y fisuras en el diseño y ejecución de estudios descriptivos. Revista Med, 23(1), 40-51.

Castillo, A. (2013). Aprendizaje y desarrollo emocional: acciones y experiencias psicoeducativas en un aula de preescolar (Thesis). Recuperado de: http://repositorio.uned.ac.cr/reuned/handle/120809/995

Delgado, L. C., Guerra, P., Perakakis, P., \& Viedma, M. I. (2010). Eficacia de un programa de entrenamiento en conciencia plena (Mindfulness) y valores humanos como herramienta de regulación emocional y prevención del estrés para profesores. Behavioral Psychology, 23. 
De Vibe, M., Bjørndal, A., Fattah, S., Dyrdal, G. M., Halland, E., \& Tanner- Smith, E. E. (2018). La práctica del Mindfulness o Atención Plena mejora la salud y la calidad de vida en adultos. Caracas: The Campbell Collaboration. Retrieved from http://scioteca.caf.com/handle/123456789/1262

Flores, L. (2016). La influencia de la relajación en la concentración de niños de cuatro a cinco años, previo a actividades de lógica-matemática. Tesis doctoral. Universidad de las Américas, Quito (Ecuador). Recuperado de: http://dspace.udla.edu.ec/handle/33000/5535

Gaeta, M. L., Pilar Teruel, M., \& Orejudo, S. (2012). Aspectos motivacionales, volitivos y metacognitivos del aprendizaje autorregulado. Electronic Journal of Research in Educational Psychology, 10(26). Recuperado de: http://www.redalyc.org/resumen.oa?id=293123551005

Gallegos, W. A. (2015). Conducta prosocial y psicología positiva. Avances en Psicología, 23(1), 37-47.

Goleman, D. (1995). Inteligencia emocional. Madrid: Editorial Kairós.

Hervás, G., Cebolla, A., \& Soler, J. (2016). Intervenciones psicológicas basadas en Mindfulness y sus beneficios: estado actual de la cuestión. Clínica y Salud, 27(3), 115-124.

Jiménez, J. E., Hernández, S., García, E., Díaz, A., Rodríguez, C., \& Martín, R. (2012). Test de atención D2: datos normativos y desarrollo evolutivo de la atención en educación primaria. European journal of education and psychology, 5(1), 93-106.

Juric, L. C., Introzzi, I., Andrés, M. L., \& Stelzer, F. (2016). La contribución de las Funciones Ejecutivas a la Autorregulación. Cuadernos de Neuropsicología / Panamerican Journal of Neuropsychology, 10(2), 106-128. Recuperado de: http://www.cnps.cl/index.php/cnps/article/view/238

Kuhl, J. (1985). Volitional Mediators of Cognition-Behavior Consistency: Self-Regulatory Processes and Action Versus State Orientation. En SSSP Springer Series in Social Psychology. Action Control: From Cognition to Behavior (pp. 101-128). 
Moctezuma, A. (2016). La música como estrategia para la educación de la inteligencia emocional en nivel preescolar. Revista Multidisciplinaria de Avances de Investigación, 2(2), 46-59.

Monteoliva, J. M., Ison, M. S., \& Pattini, A. E. (2014). Evaluación del desempeño atencional en niños: eficacia, eficiencia y rendimiento. Interdisciplinaria, 31(2). Recuperado de http://www.redalyc.org/resumen.oa?id=18032537002

Pérez, E. (2009). Desarrollo de los procesos atencionales. Universidad Complutense de Madrid, Servicio de Publicaciones, Madrid. Recuperado de https://eprints.ucm.es/8447/

Saarni, C. (1999). The development of Emotional Competence. New York: The Guilford Press.

Vives, T., Durán, C., Varela, M., \& Fortoul, T. (2014). La autorregulación en el aprendizaje, la luz de un faro en el mar. Investigación en Educación Médica, 3(9), 34-39. 